\title{
ASYMPTOTIC PROPERTIES OF THE ALGEBRAIC MOMENT RANGE PROCESS
}

\author{
HOLGER DETTE AND FABRICE GAMBOA
}

\begin{abstract}
Let $M_{n}$ denote the $n$th moment space of the set of all probability measures on the interval $[0,1], P_{n}$ the uniform distribution on the set $M_{n}$ and $r_{n+1}$ the maximal range of the $(n+1)$ th moments corresponding to a random moment point $C_{n}$ with distribution $P_{n}$ on $M_{n}$. We study several asymptotic properties of the stochastic process $\left(r_{\lfloor n t\rfloor+1}\right)_{t \in[0, T]}$ if $n \rightarrow \infty$. In particular weak convergence to a Gaussian process and a large deviation principle are established.
\end{abstract}

Key words: Moment spaces, weak convergence, large deviations, canonical moments, range of the moment space, beta-distribution.

\section{Abbreviated title: Asymptotic properties of the range process.}

\section{MSC subject classification: 60F10, 30E05}

\section{INTRODUCTION}

Moment problems have a long history in mathematics, which involves numerous subject areas, including continued fractions, orthogonal polynomials, approximation theory, probability theory and statistics [see e.g. Landau (1987)]. In this paper we will study, in some stochastic sense, the size of the moments space of probability measures on the interval $[0,1]$. To be precise let $\mathcal{P}([0,1])$ denote the set of all probability measures on the interval $[0,1]$. Further, let for $n \in \mathbb{N}^{*}, \Phi_{n}(x):=\left(x, x^{2}, \ldots, x^{n}\right)$ denote the vector of monomials up to the order $n$ and

$$
M_{n}:=\left\{\int_{0}^{1} \Phi_{n}(x) \mu(d x): \quad \mu \in \mathcal{P}([0,1])\right\} \subset \mathbb{R}^{n}
$$

be the $n$th moment space. Obviously, $M_{n}$ is a subset of the cube $[0,1]^{n}$ and it is the convex hull of the curve $\left(\Phi_{n}(x)\right)_{x \in[0,1]}$ in $\mathbb{R}^{n}$. A vector $c \in \mathbb{R}^{n}$ is an element of $M_{n}$ if, and only if, the set

$$
\mathcal{S}_{n}(c):=\left\{\mu \in \mathcal{P}([0,1]): \int_{0}^{1} \Phi_{n}(x) \mu(d x)=c\right\}
$$

is non empty. The set $M_{n}$ is a very small compact subset of the unit cube $[0,1]^{n}$ with volume

$$
\operatorname{Vol}\left(M_{n}\right)=\prod_{k=1}^{n} \frac{\Gamma(k) \Gamma(k)}{\Gamma(2 k)} \approx c \cdot 2^{-n^{2}}
$$


[see Karlin and Studden (1966), p. 129] and has a very complicated geometric structure [see Shohat and Tamarkin (1943) or Karlin and Shapeley (1953)]. As the moment space $M_{n}$ has a non void interior we may define $P_{n}$ as the uniform distribution on the set $M_{n}$. The motivation for studying a uniform distribution on the $n$th moment space stems from an attempt to obtain a better understanding of the shape and structure of the set $M_{n}$ by looking, in some sense at a typical point in the set $M_{n}$ [see Chang, Kemperman and Studden (1993), Gupta (1999), and Gamboa and Lodaza-Chang (2004)]. If $C_{n}$ is a random vector with distribution $P_{n}$ and, for $k \in\{0, \ldots, n\}, \Pi_{n}^{k}$ is the natural projection operator from $\mathbb{R}^{n}$ to $\mathbb{R}^{k}$, these authors investigate the asymptotic behaviour of the random vector $Z_{n}^{(k)}=\Pi_{k}^{n}\left(C_{n}\right)$ as $n \rightarrow \infty$ and $k$ is fixed. In particular a normal limit theorem and some large deviation princples (LDP) for functions of $Z_{n}^{(k)}$ are established in these references.

The present paper is devoted to the problem of studying random variables on the moment space $M_{n}$, where the index $k$ varies with the dimension of $M_{n}$ as $n \rightarrow \infty$. In particular we are interested in the range of the moment space $M_{n+1}$, which can be interpreted as the width of $M_{n+1}$ in the $(n+1)$ th coordinate, if the first $n$ coordinates of a "typical" point of $M_{n}$ are fixed. To be precise let for $c \in M_{n}$, denote by

$$
r_{n+1}(c):=\sup _{\mu_{1}, \mu_{2} \in \mathcal{S}_{n}(c)} \int_{0}^{1} x^{n+1} d\left(\mu_{2}-\mu_{1}\right)(x)
$$

the $(n+1)$-th moment range $r_{n+1}(c)$. We will study the asymptotic properties of the range process

$$
R_{t}^{n}:=4^{\lfloor n t\rfloor} r_{\lfloor n t\rfloor+1}\left(\Pi_{n}^{\lfloor n t\rfloor}\left(C_{n}\right)\right), \quad t \in[0, T]
$$

where $C_{n}$ is a random vector with distribution $P_{n}$ on the set $M_{n}, 0<T<1$ and $\lfloor\gamma\rfloor$ denotes the integer part of $\gamma \in \mathbb{R}$. In particular we prove a law of large numbers for the process $\left(R_{t}^{n}\right)_{t \in[0, T]}$, weak convergence of an appropriately standardized version of $\left(R_{t}^{n}\right)_{t \in[0, T]}$ to a Gaussian process, and establish a large deviation principle with a good rate function.

The remaining part of the paper is organized as follows. The main results are stated in Section 2. We will make extensive use of the canonical coordinates introduced by Skibinsky (1967), which will be the main tool for the derivation of the asymptotic properties of the process $\left(R_{t}^{n}\right)_{t \in[0, T]}$. The proofs of our main results are presented in Section 3, while some more technical arguments regarding properties of the beta distribution and gamma function are given in Section 4.

\section{Asymptotic PRoperties of the PRocess $\left(R_{t}^{n}\right)_{t \in[0, T]}$}

2.1. Preliminaries. The main tool for our investigations of the asymptotic properties of the process $\left(R_{t}^{n}\right)_{t \in[0, T]}$ is the representation of the random variable

$$
r_{k+1}\left(\Pi_{n}^{k}\left(C_{n}\right)\right), \quad(n \in \mathbb{N}, k \in\{0, \ldots, n\})
$$

as a product of independent random variables. This representation is achieved by introducing "canonical" coordinates which map the interior of the $n$th moment space $M_{n}$ in a one to one manner onto the open cube $(0,1)^{n}$ [see Skibinsky (1967)]. To be precise define for $k=2,3, \ldots$ 
and for a given point $\left(c_{1}, \ldots, c_{k-1}\right) \in M_{k-1} c_{k}^{+}=c_{k}^{+}\left(c_{1}, \ldots, c_{k-1}\right)$ and $c_{k}^{-}=c_{k}^{-}\left(c_{1}, \ldots, c_{k-1}\right)$ as the largest and smallest value of $c_{k}$ such that $\left(c_{1}, \ldots, c_{k}\right) \in M_{k}$, that is

$$
\begin{aligned}
& c_{k}^{-}=\min \left\{\int_{0}^{1} x^{k} \mu(d x) \mid \mu \in S_{k-1}\left(c_{1}, \ldots, c_{k-1}\right)\right\}, \\
& c_{k}^{+}=\max \left\{\int_{0}^{1} x^{k} \mu(d x) \mid \mu \in S_{k-1}\left(c_{1}, \ldots, c_{k-1}\right)\right\} .
\end{aligned}
$$

Note that $c_{k}^{-} \leq c_{k} \leq c_{k}^{-}$and that both inequalities are strict if and only if $\left(c_{1}, \ldots, c_{k-1}\right) \in$ $\operatorname{int} M_{k-1}$ where int $B$ denotes the interior of the set $B$ [see Skibinsky (1967)]. For a moment point $c=\left(c_{1}, \ldots, c_{n}\right)$ in the interior of $M_{n}$ the canonical moments or canonical coordinates of $c$ are defined by

$$
p_{1}=c_{1}, \text { and } p_{k}=\frac{c_{k}-c_{k}^{-}}{c_{k}^{+}-c_{k}^{-}}=\frac{c_{k}-c_{k}^{-}}{r_{k}\left(c_{1}, \ldots, c_{k-1}\right)} \quad k=2, \ldots, n
$$

Note that $0<p_{k}<1(k=1, \ldots, n)$ if $\left(c_{1}, \ldots, c_{n}\right) \in \operatorname{int} M_{n}$, and that the definition (2.2) defines a one to one mapping between $\operatorname{int} M_{n}$ and the open unit cube $(0,1)^{n}$. For more details regarding canonical moments we refer to the work of Skibinsky $(1967,1968,1969)$ and to the monograph of Dette and Studden (1997). In particular it is shown in these references that the range can be represented as

$$
r_{k+1}\left(c_{1}, \ldots, c_{k}\right)=\prod_{j=1}^{k} p_{j}\left(1-p_{j}\right), \quad(k \geq 1) .
$$

Moreover, from the representation (2.2) it is easy to see that

$$
\frac{\partial c_{k}}{\partial p_{j}}=\left\{\begin{array}{ccc}
0 & \text { if } & j>k \\
r_{k}=r_{k}\left(c_{1}, \ldots, c_{k-1}\right) & \text { if } & j=k
\end{array}\right.
$$

(with the convention $r_{1}=1$ ), and consequently the canonical moments $p_{1}^{(n)}, \ldots, p_{n}^{(n)}$ of random moment vector $C_{n}$ with a uniform distribution on $M_{n}$ have a density given by

$$
\prod_{j=1}^{n-1} \frac{\Gamma(2 n-2 j)}{(\Gamma(n-j))^{2}} \prod_{j=1}^{n} r_{j}=\prod_{j=1}^{n-1} \frac{\Gamma(2 n-2 j)}{(\Gamma(n-j))^{2}} \prod_{j=1}^{n-1}\left(p_{i}\left(1-p_{i}\right)\right)^{n-i} .
$$

In other words the $(k+1)$ th range of a random moment point $C_{n}$ can be represented as

$$
r_{k+1}\left(\Pi_{n}^{k}\left(C_{n}\right)\right)=\prod_{j=1}^{k}\left[p_{j}^{n}\left(1-p_{j}^{n}\right)\right], \quad(n \in \mathbb{N}, k \in\{0, \ldots, n\}),
$$

where $\left(p_{j}^{n}\right)_{n \in \mathbb{N}, 1 \leq j \leq n}$ is a triangular array of independent random variables and for $j=$ $1, \ldots, n-1$ the random variable $p_{j}^{n}$ follows a $\beta(n-j, n-j)$ distribution. The representation (2.1) will be essential for our investigations of the asymptotic properties of the range process in the following discussion. In the whole paper, we take the convention that $\log \tau=-\infty$ whenever $\tau \leq 0$ and we denote the set of all continuous functions on $[0, T]$ by $C([0, T])$. 
2.2. Law of large numbers and weak convergence. In the present and next sections, $\left(R_{t}^{n}\right)_{t \in[0, T]}$ will be considered as a process with c.a.d-l.a.g. pathes. Thus, we consider convergence in Skorohod topology [see [Billingsley(1999)]]. Moreover, we will denote by the symbol $\rightsquigarrow$ the convergence in distribution.

Theorem 2.1. For $0<T<1,\left(R_{t}^{n}\right)_{t \in[0, T]}$ converges in probability towards the deterministic process $(\sqrt{1-t})_{t \in[0, T]}$.

Theorem 2.2. Let $\left(B_{t}\right)_{t \in[0, T]}$ denotes the standard Brownian motion, then for any $0<T<1$ we have

$$
\left(\left(\frac{R_{t}^{n}}{\sqrt{1-t}}\right)^{\sqrt{n}}\right)_{t \in[0, T]} \rightsquigarrow\left(\exp \frac{1}{\sqrt{2}} \int_{0}^{t} \frac{d B_{u}}{1-u}\right)_{t \in[0, T]} .
$$

2.3. Large deviations. Recall (see for example [Dembo and Zeitouni(1998)]) that a sequence $\left(Q_{n}\right)$ of probability measures on a measurable Hausdorff space $(U, \mathcal{B}(U))$ satisfies a large deviation principle (LDP) with rate function $I$ if

i) $I$ is lower semicontinuous (lsc), with values in $\mathbb{R}^{+} \cup\{+\infty\}$.

ii) For any measurable set $A$ of $U$ :

$$
-I(\operatorname{Int} A) \leq \liminf _{n \rightarrow \infty} \frac{1}{n} \log Q_{n}(A) \leq \limsup _{n \rightarrow \infty} \frac{1}{n} \log Q_{n}(A) \leq-I(\operatorname{Clo} A)
$$

where $I(A)=\inf _{\xi \in A} I(\xi)$ and $\operatorname{Int} A$ (resp. Clo $A$ ) is the interior (resp. the closure) of A.

Moreover, the rate function $I$ is called good if its level sets $\{x \in U: I(x) \leq a\}$ are compact for any $a \geq 0$. More generally, a sequence of $U$-valued random variables is said to satisfy a LDP if their distributions satisfy a LDP.

In order to make this paper self-contained let us recall some known facts and tools of the theory of large deviations which will be useful in the paper (we refer to [Dembo and Zeitouni(1998)] for more results regarding large deviations). In particular we will use the following two concepts throughout this paper.

- Contraction principle. Assume that $\left(Q_{n}\right)$ satisfies an LDP on $(U, \mathcal{B}(U))$ with good rate function $I$. Let $T$ be a continuous mapping from $U$ to another space $V$. Then, $\left(Q_{n} \circ T^{-1}\right)$ satisfies an LDP on $(V, \mathcal{B}(V))$ with good rate function

$$
I^{\prime}(y)=\inf _{x: T(x)=y} I(x), \quad(y \in V)
$$

- Exponential approximation. Assume that $U$ is a metric space and let $d$ denotes the metric on $U$. Let $\left(X_{n}\right)_{n \in \mathbb{N}}$ be a $U$-valued random sequence satisfying an LDP with good rate function $I$. Let $\left(Y_{n}\right)_{n \in \mathbb{N}}$ be another $U$-valued random sequence. If for any $\xi>0$

$$
\limsup _{n \rightarrow \infty} \frac{1}{n} \log \mathbb{P}\left(d\left(X_{n}, Y_{n}\right)>\xi\right)=-\infty,
$$

then $\left(Y_{n}\right)_{n \in \mathbb{N}}$ shares the same LDP as $\left(X_{n}\right)_{n \in \mathbb{N}}$. 
In the following let $B V([0, T])$ denote the set of all real valued functions of bounded variation on the interval $[0, T]$. We identify $B V([0, T])$ with the set $\mathcal{M}([0, T])$ of all real measures on $[0, T]$. Indeed, if $f$ is an element of the set $B V([0, T])$, there exists a unique measure $\mu_{f} \in \mathcal{M}([0, T])$ with

$$
f(u)=\int_{0}^{u} \mu_{f}(d t)=\int_{0}^{u} \mu_{f}^{+}(d t)-\int_{0}^{u} \mu_{f}^{-}(d t),
$$

where, for $\mu \in \mathcal{M}([0, T]), \mu=\mu^{+}-\mu^{-}$is the Jordan decomposition of the measure $\mu$. The Lebesgue decomposition of the measure $\mu$ with respect to the Lebesgue measure will be denoted as

$$
\mu(d u)=h_{\mu}(u) d u+\sigma_{\mu}(d u) .
$$

Up to the identification of $B V([0, T])$ by $\mathcal{M}([0, T]), C([0, T])$ is the topological dual of $B V([0, T])$. Thus, we endow $B V([0, T])$ with the weak-* topology (duality $(B V([0, T], C([0, T]))$. Let

$$
B V_{0}^{+}([0, T])=\{f \in B V([0, T]): f(0)=0, f \text { non decreasing on }[0, T]\}
$$

and

$$
B V_{1}^{-}([0, T])=\{f \in B V([0, T]): f(0)=1, f>0, f \text { non increasing on }[0, T]\} .
$$

Theorem 2.3. For any $0<T<1$, the process $\left(R_{t}^{n}\right)_{t \in[0, T]}$ satisfies an $L D P$ on $B V_{1}^{-}([0, T])$ with rate function $I_{1} . I_{1}$ is a good rate function which is only finite for functions $f \in$ $B V_{1}^{-}([0, T])$. Indeed, for $f \in B V_{1}^{-}([0, T])$ the rate function has the representation

$$
\begin{aligned}
I_{1}(f) & :=-\frac{1}{2} \int_{0}^{T} \log \left[2(1-u) h_{\mu_{\log (1 / f)}}(u)\right] d u \\
& +\int_{0}^{T}(1-u) \mu_{\log (1 / f)}(d u)-\frac{T}{2} .
\end{aligned}
$$

Using the change of variable formula for Stieljes integrals (see for example [Protter (2004)] Theorem 54), we obtain the following Proposition.

Proposition 2.4. Let $f \in B V_{1}^{-}([0, T])$ and write

$$
\mu_{f}(d u)=-\mu_{f}^{d}(d u)+\delta_{0}(d u)-\sum_{j \in A_{f}} a_{j}^{f} \delta_{t_{j}^{f}}(d u),
$$

where $\mu_{f}^{d}$ is a diffuse positive measure and the measure $\mu_{f}$ has jumps at the positive points $\left(t_{j}^{f}\right)_{j \in A_{f}}$. Then,

$$
\log \left(\frac{1}{f(t)}\right)=\int_{0}^{t} \frac{\mu_{f}^{d}(d u)}{f(u)}+\sum_{j \in A_{f}: t_{j}^{f} \leq t} \log \left(1+\frac{a_{j}^{f}}{f\left(t_{j}^{f}\right)}\right) .
$$


Moreover,

$$
\begin{aligned}
I_{1}(f) & =-\frac{1}{2} \int_{0}^{T} \log \left[2(1-u) h_{\mu_{f}}(u)\right] d u+\frac{1}{2} \int_{0}^{T} \log f(u) d u \\
& +\int_{0}^{T}(1-u) \frac{\mu_{f}^{d}(d u)}{f(u)}+\sum_{j \in A_{f}}\left[\log \left(1+\frac{a_{j}^{f}}{f\left(t_{j}^{f}\right)}\right)\left(1-t_{j}^{f}\right)\right]-\frac{T}{2} .
\end{aligned}
$$

\section{Proofs}

3.1. Proof of Theorem 2.2. We will prove the theorem by showing the following propostion.

Proposition 3.1. For any $0<T<1$,

$$
\left(\sqrt{n}\left(\log R_{t}^{n}-\log \sqrt{1-t}\right)\right)_{t \in[0, T]} \rightsquigarrow\left(\frac{1}{\sqrt{2}} \int_{0}^{t} \frac{d B_{u}}{1-u}\right)_{t \in[0, T]},
$$

where $\left(B_{u}\right)_{u \in[0,1]}$ denotes a standard Brownian motion.

Proof. From (1.2) and (2.6), we may write, for $t \in[0, T]$,

$$
\begin{aligned}
\sqrt{n}\left(\log R_{t}^{n}-\log \sqrt{1-t}\right) & =\sqrt{n}\left(\sum_{j=1}^{\lfloor n t\rfloor} \log \left[p_{j}^{n}\left(1-p_{j}^{n}\right)\right]-\frac{1}{2} \log (1-t)+2\lfloor n t\rfloor \log 2\right) \\
& =\sqrt{n} \sum_{j=1}^{\lfloor n t\rfloor}\left(\rho_{j}^{n}-\mathbb{E}\left[\rho_{j}^{n}\right]\right)+A_{n}(t)
\end{aligned}
$$

where

$$
\rho_{j}^{n}=\log \left[p_{j}^{n}\left(1-p_{j}^{n}\right)\right]
$$

and the random variable $A_{n}(t)$ is defined by

$$
A_{n}(t)=\sqrt{n}\left(\sum_{j=1}^{\lfloor n t\rfloor} \mathbb{E}\left[\rho_{j}^{n}\right]-\frac{1}{2} \log (1-t)+2\lfloor n t\rfloor \log 2\right) .
$$

First, notice that as the function $(1-t)^{-\alpha}, \quad(\alpha>0)$ is increasing on the interval $[0, T]$, we have for any $t \in[0, T]$

$$
\frac{1}{n} \sum_{j=1}^{\lfloor n t\rfloor} \frac{1}{\left(1-\frac{j-1}{n}\right)^{\alpha}} \leq \int_{0}^{T} \frac{d u}{(1-u)^{\alpha}}:=\mathcal{I}_{\alpha} .
$$

In a first step we show that the process $A_{n}(t)$ converges to 0 , uniformly on the interval $[0, T]$. Indeed, using inequality (4.12) in the Appendix and the monotonicity properties of 
the functions $(1-x)^{-1}$ and $(1-x)^{-2}$ we obtain

$$
\begin{aligned}
\left|A_{n}(t)\right| & \leq \sqrt{n} C_{1} \sum_{j=1}^{\lfloor n t\rfloor} \frac{1}{(n-j+1)^{2}}+\frac{\sqrt{n}}{2}\left|\sum_{j=1}^{\lfloor n t\rfloor} \frac{1}{n-j+1}-\int_{0}^{t} \frac{d u}{1-u}\right| \\
& \leq \frac{C_{1}}{n^{3 / 2}} \sum_{j=1}^{\lfloor n T\rfloor} \frac{1}{\left(1-\frac{j-1}{n}\right)^{2}}+\frac{\sqrt{n}}{2}\left(\int_{0}^{T} \frac{d u}{1-u}-\frac{1}{n} \sum_{j=1}^{\lfloor n T\rfloor} \frac{1}{1-\frac{j-1}{n}}\right) \\
& \leq \frac{1}{\sqrt{n}}\left(C_{1} \mathcal{I}_{2}+(1-T)^{-1}\left(T+2(1-T)^{-1}\right)\right)
\end{aligned}
$$

for some constants $C_{1}, \tau_{2}>0$. This implies that $A_{n}$ converges uniformly to 0 on the interval $[0, T]$.

We will now show that the process defined in (3.1) converges to the limit given in the proposition. To obtain this result, we will use the general martingale theorem stated in [Dacunha-Castelle and Duflo (1993)] (Theorem 7.4.28 p. 226). In order to use this general result, we have to check the following three assumptions:

(A1) For all $\varepsilon>0$,

$$
\lim _{n \rightarrow \infty} \sum_{j=1}^{\lfloor n T\rfloor} \mathbb{P}\left(\left|\tilde{\rho}_{j}^{n}\right| \geq \varepsilon\right)=0 .
$$

(A2) There exists an $\varepsilon>0$, such that for all $t \in[0, T]$,

$$
\lim _{n \rightarrow \infty} \sum_{j=1}^{\lfloor n t\rfloor} \mathbb{E}\left(\mathbb{I}_{\left\{\left|\tilde{\rho}_{j}^{n}\right|<\varepsilon\right\}} \tilde{\rho}_{j}^{n}\right)=0 .
$$

(A3) There exists an $\varepsilon>0$, such that for all $t \in[0, T]$,

$$
\lim _{n \rightarrow \infty} \sum_{j=1}^{\lfloor n t\rfloor} \operatorname{Var}\left(\mathbb{I}_{\left\{\left|\tilde{\rho}_{j}^{n}\right|<\varepsilon\right\}} \tilde{\rho}_{j}^{n}\right)=\int_{0}^{t} \frac{d u}{2(1-u)^{2}}=\frac{t}{2(1-t)}
$$

where we used the notation $\tilde{\rho}_{j}^{n}=\sqrt{n}\left(\rho_{j}^{n}-\mathbb{E}\left[\rho_{j}^{n}\right]\right)$ and $\mathbb{I}_{A}$ denotes the indicator function of the set $A$.

Let us first check that the assumption (A1) holds. Using Markov's inequality and the estimate (4.16) in the Appendix for the fourth moment of the centered random variable $\tilde{\rho}_{j}^{n}$ we may write for $\varepsilon>0, n \in \mathbb{N}^{*}$ and $j \in\{1, \ldots, n\}$

$$
\mathbb{P}\left(\left|\tilde{\rho}_{j}^{n}\right| \geq \varepsilon\right) \leq \frac{n^{2}}{\varepsilon^{4}} \mathbb{E}\left(\rho_{j}^{n}-\mathbb{E}\left[\rho_{j}^{n}\right]\right)^{4} \leq \frac{n^{2} C_{3}}{\varepsilon^{4}(n-j+1)^{4}}
$$

with some positive constant $C_{3}$. This gives

$$
\sum_{j=1}^{\lfloor n T\rfloor} \mathbb{P}\left(\left|\tilde{\rho}_{j}^{n}\right| \geq \varepsilon\right) \leq \frac{C_{3} \mathcal{I}_{4}}{n \varepsilon^{4}}
$$


and assumption (A1) follows.

To prove assumption (A2) notice that the random variables $\tilde{\rho}_{j}^{n}$ are centered and therefore it is sufficient to show that for one $\varepsilon>0$,

$$
\lim _{n \rightarrow \infty} \sum_{j=1}^{\lfloor n T\rfloor} \mathbb{E}\left(\mathbb{I}_{\left\{\left|\tilde{\rho}_{j}^{n}\right| \geq \varepsilon\right\}}\left|\tilde{\rho}_{j}^{n}\right|\right)=0
$$

Now, using Cauchy Schwartz inequality, the bound (3.5) and the estimate (4.14) in the Appendix yields

$$
\mathbb{E}\left(\mathbb{I}_{\left\{\left|\tilde{\rho}_{j}^{n}\right| \geq \varepsilon\right\}}\left|\tilde{\rho}_{j}^{n}\right|\right) \leq \sqrt{n \operatorname{Var} \rho_{j}^{n}} \sqrt{\mathbb{P}\left(\tilde{\rho}_{j}^{n} \geq \varepsilon\right)} \leq \frac{\sqrt{C_{2} C_{3}}}{n^{3 / 2} \varepsilon^{2}\left(1-\frac{j-1}{n}\right)^{3}}
$$

with positive constants $C_{2}, C_{3}$. Therefore we obtain

$$
\sum_{j=1}^{\lfloor n T\rfloor} \mathbb{E}\left(\mathbb{I}_{\left\{\left|\tilde{\rho}_{j}^{n}\right| \geq \varepsilon\right\}}\left|\tilde{\rho}_{j}^{n}\right|\right) \leq \frac{\sqrt{C_{2} C_{3}} \mathcal{I}_{3}}{n^{1 / 2}}
$$

and assumption (A2) follows.

To show the remaining property (A3), first notice that, for $t \in[0, T]$, using the estimate (4.13) in the Appendix for the variance of $\tilde{\rho}_{j}^{n}$, we can mimic the derivation of the inequality (3.4) to easily obtain

$$
\lim _{n \rightarrow \infty} \sum_{j=1}^{\lfloor n t\rfloor} \operatorname{Var} \tilde{\rho}_{j}^{n}=\frac{t}{2(1-t)}
$$

Now,

$$
\operatorname{Var} \tilde{\rho}_{j}^{n}=\operatorname{Var}\left(\tilde{\rho}_{j}^{n} \mathbb{I}_{\left\{\left|\tilde{\rho}_{j}^{n}\right|<\varepsilon\right\}}\right)+\mathbb{E}\left(\left(\tilde{\rho}_{j}^{n}\right)^{2} \mathbb{I}_{\left\{\left|\tilde{\rho}_{j}^{n}\right| \geq \varepsilon\right\}}\right)+\left[\mathbb{E}\left(\tilde{\rho}_{j}^{n} \mathbb{I}_{\left\{\left|\tilde{\rho}_{j}^{n}\right| \geq \varepsilon\right\}}\right)\right]^{2}
$$

On the one hand, using once more the Cauchy Schwartz inequality and the estimate (4.14) and (4.16) of the Appendix we may easily show that

$$
\lim _{n \rightarrow \infty} \sum_{j=1}^{\lfloor n T\rfloor} \mathbb{E}\left(\left(\tilde{\rho}_{j}^{n}\right)^{2} \mathbb{I}_{\left\{\left|\tilde{\rho}_{j}^{n}\right| \geq \varepsilon\right\}}\right)=0
$$

On the other hand, this also implies

$$
\lim _{n \rightarrow \infty} \sum_{j=1}^{\lfloor n T\rfloor}\left[\mathbb{E}\left(\tilde{\rho}_{j}^{n} \mathbb{I}_{\left\{\left|\tilde{\rho}_{j}^{n}\right| \geq \varepsilon\right\}}\right)\right]^{2}=0
$$

and assumption (A3) holds, which completes the proof of the Proposition. 
3.2. Proof of Theorem 2.3. To prove Theorem 2.3, we will first show that an LDP holds for the following positive random measure on the interval $[0, T](0<T<1)$

$$
\nu_{n}(d x):=-\sum_{j=1}^{\lfloor n T\rfloor}\left(\xi_{n-j}+2 \log 2\right) \delta_{j / n}(d x),
$$

where $\delta_{y}$ denotes the Dirac measure at the point $y, \xi_{1}, \ldots, \xi_{n}$ are independent random variables distributed such that $\xi_{i}=\log \left(x_{i}\left(1-x_{i}\right)\right)$ and $x_{i}$ has a $\beta(i, i)$ distribution. Indeed, endowing the set $\mathcal{M}_{+}([0, T])$ of all positive measures on the interval $[0, T]$ with the weak topology, we have the following theorem.

Theorem 3.2. The sequence of random measures $\left(\nu_{n}\right)$ satisfies an LDP with a good convex rate function given by

$$
I_{2}(\mu)=-\frac{1}{2} \int_{0}^{T} \log \left[2(1-u) h_{\mu}(u)\right] d u+\int_{0}^{T}(1-u) \mu(d u)-\frac{T}{2}, \quad\left(\mu \in \mathcal{M}_{+}([0, T]),\right.
$$

Proof. The idea of the proof is to check that the assumptions of the abstract Gärtner-Ellis theorem are fulfilled (see theorem 4.5.20 in [Dembo and Zeitouni(1998)], [Gamboa et al(1999)], [Gamboa and Gassiat(1997)] and $[\operatorname{Najim}(2002)])$. For this let $f \in C([0, T])$, we will first compute the normalized cumulant generating function of $\nu_{n}$ at point $f$ that is

$$
\begin{aligned}
\Lambda_{n}(f) & :=\frac{1}{n} \log \mathbb{E}\left(\exp \left[n \nu_{n}(f)\right]\right) \\
& =\frac{1}{n} \log \mathbb{E}\left(\prod_{j=1}^{\lfloor n T\rfloor} \exp \left[-n f(j / n)\left(\xi_{n-j}+2 \log 2\right)\right]\right) \\
& =\frac{1}{n} \sum_{j=1}^{\lfloor n T\rfloor}\left[\psi_{n-j}(-n f[j / n])-2 n f(j / n) \log 2\right] .
\end{aligned}
$$

where $\psi_{n-j}(t)=\log E\left[e^{t \xi_{n-j}}\right]$ denotes the cumulant generating function of the random variable $\xi_{n-j}$. Now, using the representation (4.2) for the function $\psi_{k}(t)$ in the Appendix, we obtain:

- $\underline{\text { First case }}$

$$
\sup _{u \in[0, T]} \frac{f(u)}{1-u}<1
$$

For $n$ large enough, $\Lambda_{n}(f)$ is finite and using the representation (4.4) in the Appendix it follows that

$$
\begin{aligned}
\Lambda_{n}(f) & =\frac{1}{n} \sum_{j=1}^{\lfloor n T\rfloor}\left\{-\frac{1}{2} \log \left(1-\frac{n f(j / n)}{n-j+1}\right)\right. \\
& +4\left[\varphi_{0}(n-j+1-n f[j / n])-\varphi_{0}(n-j+1)\right] \\
& \left.-2\left[\varphi_{0}[2(n-j+1-n f[j / n])]-\varphi_{0}(2 n-2 j+2)\right]\right\},
\end{aligned}
$$


where the function $\varphi_{0}$ is defined by

$$
\varphi_{0}(z):=\int_{0}^{+\infty} \frac{\arctan \left(\frac{t}{z}\right)}{\exp (2 \pi t)-1} d t .
$$

By Lemma 4.1 in the Appendix we therefore obtain the estimate

$$
\left|\Lambda_{n}(f)+\frac{1}{n} \sum_{j=1}^{\lfloor n T\rfloor} \frac{1}{2} \log \left(1-\frac{n f(j / n)}{n-j+1}\right)\right| \leq \frac{5 \mathcal{I}_{0}}{n^{2}} \sum_{j=1}^{\lfloor n T\rfloor} \frac{|f(j / n)|}{\left[\left(1-\frac{j-1}{n}-f(j / n)\right) \wedge\left(1-\frac{j-1}{n}\right)\right]^{2}},
$$

where $a \wedge b$ denotes the minimum of $a$ and $b$. This last inequality allows to conclude that

$$
\lim _{n \rightarrow \infty} \Lambda_{n}(f)=-\frac{1}{2} \int_{0}^{T} \log \left(1-\frac{f(u)}{1-u}\right) d u .
$$

- $\underline{\text { Second case }}$

$$
\sup _{u \in[0, T]} \frac{f(u)}{1-u}>1 .
$$

As $f$ is continuous, for $n$ large enough, at least one of the summand in (3.10) is infinite. Hence

$$
\lim _{n \rightarrow \infty} \Lambda_{n}(f)=+\infty .
$$

- Third case $\sup _{u \in[0, T]} \frac{f(u)}{1-u}=1$.

We do not know in general what happens in this case. But, as in the proof of theorem 4 in [Gamboa et al(1999)] it does not matter.

Now, for $f \in C([0, T])$, let

$$
\Lambda(f):=-\frac{1}{2} \int_{0}^{T} \log \left(1-\frac{f(u)}{1-u}\right) d u .
$$

Further, for $\mu \in \mathcal{M}_{+}([0, T])$, let

$$
\Lambda^{*}(\mu):=\sup _{f \in C([0, T])}\left[\int_{0}^{T} f(u) \mu(d u)-\Lambda(f)\right] .
$$

Making the change of variable $g(\cdot)=f(\cdot) /(1-\cdot)$ in the last equation and using the duality results of Section 3 in [Borwein and Lewis(1993)] we may deduce, that for any $\mu \in \mathcal{M}_{+}([0, T])$

$$
\Lambda^{*}(\mu)=I_{2}(\mu) \text {. }
$$

The remaining part of the proof now follows by exactly the same arguments as given in [Gamboa et al(1999)] where the authors use a variant of the Gärtner-Ellis-Baldi's theorem (see [Dembo and Zeitouni(1998)] p. 157). We just give the skeleton of the proof and refer to [Gamboa et al(1999)] for more details.

The upper bound is proved in a classical way by first showing that it holds for compact sets of $\mathcal{M}_{+}([0, T])$. This is a direct consequence of Theorem 4.5.3 b) of [Dembo and Zeitouni(1998)]. To enlarge this upper bound to non compact sets, we use the following lemma that sets the exponential tightness of the sequence $\left(\nu_{n}\right)$. 


\section{Lemma 3.3.}

$$
\lim _{a \rightarrow+\infty} \limsup _{n \rightarrow \infty} \frac{1}{n} \log \mathbb{P}\left(\sup _{f \in C([0, T]):\|f\|_{\infty}=1} \int_{[0, T]} f(x) \nu_{n}(d x)>a\right)=-\infty .
$$

Proof. Obviously

$$
\left\{\sup _{f \in C([0, T]):\|f\|_{\infty}=1} \int_{[0, T]} f(x) \nu_{n}(d x)>a\right\} \subset\left\{-\sum_{j=1}^{\lfloor n T\rfloor}\left(\xi_{n-j}+2 \log 2\right)>a\right\} .
$$

Furthermore Markov exponential inequality and the limit in (3.12) give, for $\theta<1-T$

$$
\begin{aligned}
& \limsup _{n \rightarrow \infty} \frac{1}{n} \log \mathbb{P}\left(-\sum_{j=1}^{\lfloor n T\rfloor}\left(\xi_{n-j}+2 \log 2\right)>a\right) \leq \\
&-\theta a+\frac{1}{2}[(1-\theta-T) \log (1-\theta-T)-(1-T) \log (1-T)-(1-\theta) \log (1-\theta)] .
\end{aligned}
$$

Optimizing the last bound with respect to $\theta<1-T$ we finally obatin

$$
\begin{aligned}
& \limsup _{n \rightarrow \infty} \frac{1}{n} \log \mathbb{P}\left(-\sum_{j=1}^{\lfloor n T\rfloor}\left(\xi_{n-j}+2 \log 2\right)>a\right) \leq \\
& -a \frac{1+(T-1) e^{2 a}}{1-e^{2 a}}+\frac{1}{2}\left[\frac{-T+2(T-1) e^{2 a}}{1-e^{2 a}} \log \frac{-T+2(T-1) e^{2 a}}{1-e^{2 a}}\right. \\
& \left.\quad-(1-T) \log (1-T)-\frac{(T-2) e^{2 a}}{1-e^{2 a}} \log \frac{(T-2) e^{2 a}}{1-e^{2 a}}\right]
\end{aligned}
$$

which yields to the result.

The lower bound is more technical. As usual, it is performed using the tilted probability technique. Here, the tilted probability depends on a function $f_{\mu}$ associated to the measure $\mu$ on which we recenter the process. There are essentially two steps. In the first one we show that there exist nice measures for which the associated functions exist and fall in the first case of the previous discussion about the limit of the function $\Lambda_{n}$. Roughtly speaking, this is equivalent to the fact that the supremum of the function $\Lambda^{*}$ is attained for $f_{\mu}$. In the second step we show that these nice measures are dense in the space $\mathcal{M}_{+}([0, T])$.

By the same arguments as given in the proof of Theorem 4.1 in [Najim(2002)] we obtain from the last theorem and the contraction principle the following corollary.

Corollary 3.4. The process $\left(-\log R_{t}^{n}\right)_{t \in[0, T]}$ satisfies an LDP in $B V_{0}^{+}([0, T])$ with a good rate function given by

$$
I_{3}(f):=-\frac{1}{2} \int_{0}^{T} \log \left[2(1-u) h_{\mu_{f}}(u)\right] d u+\int_{0}^{T}(1-u) \mu_{f}(d u)-\frac{T}{2}, \quad\left(f \in B V_{0}^{+}([0, T])\right)
$$

where the function $h_{\mu}$ has been defined by (2.8). 
Further the mapping $W$ from $B V_{0}^{+}([0, T])$ to $B V_{1}^{-}([0, T])$ defined by

$$
W(f)=\exp (-f),\left(f \in B V_{0}^{+}([0, T])\right)
$$

is continuous. Indeed, $\exp (-f)$ has jumps at the same points as the function $f$ and the weak convergence is equivalent to the pointwise convergence on set of continuity points of the limit. Hence, the proof of Theorem 2.3 follows from the contraction principle and the last Corollary.

\section{Appendix: Around the $\beta$ Distribution}

Let $\alpha, \beta>-1$, recall that the distribution $\beta(\alpha, \beta)$ is the probability measure on the interval $[0,1]$ with density

$$
f_{\alpha, \beta}(x)=\frac{x^{\beta}(1-x)^{\alpha}}{B(\beta+1, \alpha+1)},(x \in[0,1]),
$$

where the Beta integral $B$ may be expressed by the way of the Gamma function:

$$
B(p, q)=\int_{0}^{1} x^{p-1}(1-x)^{q-1} d x=\frac{\Gamma(p) \Gamma(q)}{\Gamma(p+q)}, \quad(p, q>0) .
$$

For any integer $k$, let $\iota_{k}$ be a random variable with distribution $\beta(k, k)$ and set

$$
\xi_{k}=\log \left[\iota_{k}\left(1-\iota_{k}\right)\right]=\log \iota_{k}+\log \left(1-\iota_{k}\right) .
$$

As $4 \exp \xi_{k} \sim \beta\left(-\frac{1}{2}, k\right)$, for $\tau \in \mathbb{R}$, the cumulant generating function of $\xi_{k}$ is given by

$$
\psi_{k}(\tau)=\log \mathbb{E}\left[\exp \left(\tau \xi_{k}\right)\right]= \begin{cases}2 \log \Gamma(\tau+k+1)+\log \Gamma(2 k+2) & \\ -\log \Gamma(2 \tau+2 k+2)-2 \log \Gamma(k+1) & \text { if } \tau>-1-k \\ +\infty & \text { otherwise. }\end{cases}
$$

For $z \in \mathbb{C}$ with $\Re(z)>0$, Binet's second formula (see for [Abramowitz and Stegun (1964)]), gives an alternative expression for the $\log \Gamma$ function, that is

$$
\log \Gamma(z)=\left(z-\frac{1}{2}\right) \log z-z+\frac{1}{2} \log 2 \pi+2 \varphi_{0}(z),
$$

where the function $\varphi_{0}$ is defined by

$$
\varphi_{0}(z):=\int_{0}^{+\infty} \frac{\arctan \left(\frac{t}{z}\right)}{\exp (2 \pi t)-1} d t .
$$

Using this representation we may rewrite, for $\tau>-1-k$

$$
\begin{aligned}
\psi_{k}(\tau) & =-\frac{1}{2} \log \left(1+\frac{\tau}{k+1}\right)-2 \tau \log 2 \\
& +4\left[\varphi_{0}(\tau+k+1)-\varphi_{0}(k+1)\right] \\
& +2\left[\varphi_{0}(2 k+2)-\varphi_{0}(2 \tau+2 k+2)\right] .
\end{aligned}
$$


Moreover, the first derivative of $\psi_{k}$ can be represented as

$$
\begin{aligned}
\psi_{k}^{\prime}(\tau) & =-\frac{1}{2(k+1+\tau)}-2 \log 2 \\
& +4\left[\varphi_{0}^{\prime}(\tau+k+1)-\varphi_{0}^{\prime}(2 \tau+2 k+2)\right]
\end{aligned}
$$

and, for $l \geq 2$ integer, the derivative of order $l$ of $\psi_{k}^{(l)}$ is

$$
\psi_{k}^{(l)}(\tau)=\frac{(-1)^{l}(l-1) !}{2(k+1+\tau)^{l}}+4 \varphi_{0}^{(l)}(\tau+k+1)-2^{l+1} \varphi_{0}^{(l)}(2 \tau+2 k+2)
$$

From the last equations we may deduce

$$
\begin{aligned}
\mathbb{E}\left(\xi_{k}\right)= & \psi_{k}^{\prime}(0)=-\frac{1}{2(k+1)}-2 \log 2 \\
& +4\left[\varphi_{0}^{\prime}(k+1)-\varphi_{0}^{\prime}(2 k+2)\right] \\
\operatorname{Var}\left(\xi_{k}\right)= & \psi_{k}^{\prime \prime}(0)=\frac{1}{2(k+1)^{2}}+4\left[\varphi_{0}^{\prime \prime}(k+1)-2 \varphi_{0}^{\prime \prime}(2 k+2)\right], \\
\mathbb{E}\left[\xi_{k}-\mathbb{E}\left(\xi_{k}\right)\right]^{4}= & \psi_{k}^{(4)}(0)+3\left[\psi_{k}^{\prime \prime}(0)\right]^{2}=\frac{15}{4(k+1)^{4}}+4 \varphi_{0}^{(4)}(k+1) \\
& -32 \varphi_{0}^{(4)}(2 k+2)+\frac{12\left(\varphi_{0}^{\prime \prime}(k+1)-2 \varphi_{0}^{\prime \prime}(2 k+2)\right)}{(k+1)^{2}} \\
& +48\left[\varphi_{0}^{\prime \prime}(k+1)-2 \varphi_{0}^{\prime \prime}(2 k+2)\right]^{2} .
\end{aligned}
$$

Further, we have the following obvious lemma.

Lemma 4.1. For $\alpha>0$ and $\eta>-\alpha$

$$
\begin{array}{r}
\left|\varphi_{0}(\alpha+\eta)-\varphi_{0}(\alpha)\right| \leq \frac{|\eta|}{(\alpha \wedge(\alpha+\eta))^{2}} \mathcal{I}_{0} \\
\left|\varphi_{0}^{\prime}(\alpha)\right| \leq \frac{\mathcal{I}_{0}}{\alpha^{2}}, \quad\left|\varphi_{0}^{(2)}(\alpha)\right| \leq \frac{2 \mathcal{I}_{0}}{\alpha^{3}} \quad \text { and } \quad\left|\varphi_{0}^{(4)}(\alpha)\right| \leq \frac{72 \mathcal{I}_{0}}{\alpha^{5}}
\end{array}
$$

with

$$
\mathcal{I}_{0}=\int_{0}^{+\infty} \frac{t}{\exp (2 \pi t)-1} d t
$$


Using the previous lemma and equations (4.7), (4.8) and (4.9), we obtain the following bounds for the moments of the random variables $\xi_{k}$

$$
\begin{aligned}
& \left|\mathbb{E}\left(\xi_{k}\right)-\left(-\frac{1}{2(k+1)}-2 \log 2\right)\right| \leq \frac{5 \mathcal{I}_{0}}{(k+1)^{2}}:=\frac{C_{1}}{(k+1)^{2}} \\
& \left|\operatorname{Var}\left(\xi_{k}\right)-\frac{1}{2(k+1)^{2}}\right| \leq \frac{10 \mathcal{I}_{0}}{(k+1)^{3}}=\frac{2 C_{1}}{(k+1)^{3}}, \\
& \operatorname{Var}\left(\xi_{k}\right) \leq \frac{1}{2(k+1)^{2}}+\frac{10 \mathcal{I}_{0}}{(k+1)^{3}} \leq \frac{1 / 2+10 \mathcal{I}_{0}}{(k+1)^{2}} \leq \frac{C_{2}}{(k+1)^{2}} \\
& \mathbb{E}\left[\xi_{k}-\mathbb{E}\left(\xi_{k}\right)\right]^{4} \\
& \quad \leq \frac{15}{4(k+1)^{4}}+\frac{319 \mathcal{I}_{0}}{(k+1)^{5}}+\frac{300 \mathcal{I}_{0}^{2}}{(k+1)^{6}} \\
& \quad \leq \frac{15 / 4+319 \mathcal{I}_{0}+300 \mathcal{I}_{0}^{2}}{(k+1)^{4}} \leq \frac{C_{3}}{(k+1)^{4}}
\end{aligned}
$$

with positive constants $C_{1}, C_{2}, C_{3}$.

Acknowledgements. The support of the Deutsche Forschungsgemeinschaft (SFB 475, "Komplexitätsreduktion in multivariaten Datenstrukturen") is gratefully acknowledged. Parts of the paper were written during the first author's visit at the Laboratoire de Statistiques et de Probabilités of the Université Paul Sabatier in Toulouse, and this author would like to thank the institute for its hospitality. The authors are also grateful to Isolde Gottschlich, who typed parts of this paper with considerable technical expertise.

\section{REFERENCES}

[Abramowitz and Stegun (1964)] Abramowitz, M. and Stegun, I. A. (1964). Handbook of mathematical functions with formulas, graphs, and mathematical tables.. National Bureau of Standards Applied Mathematics Series, 55

[Billingsley(1999)] Billingsley, P. (1999). Convergence of probability measures. John Wiley \& Sons Inc., New York, second edition. ISBN 0-471-19745-9. A Wiley-Interscience Publication.

[Borwein and Lewis(1993)] Borwein, J. M. and Lewis, A. S. (1993). Partially-finite programming in $L_{1}$ and the existence of maximum entropy estimates. SIAM J. Optim., 3(2):248-267. ISSN 1052-6234.

[Bretagnolle(1979)] Bretagnolle, J. (1979). Formule de Chernoff pour les lois empiriques de variables a valeurs dans des espaces generaux. Asterisque, 68:32-52.

[Chang et al.(1993) Chang, Kemperman, and Studden] Chang, F. C., Kemperman, J. H. B. and Studden, W. J. (1993). A normal limit theorem for moment sequences. Ann. Probab., 21(3):1295-1309. ISSN 0091-1798.

[Dembo and Zeitouni(1998)] Dembo, A. and Zeitouni, O. (1998). Large deviations techniques and applications. Springer-Verlag, New York, second edition ISBN 0-387-98406-2.

[Dacunha-Castelle and Duflo (1993)] Dacunha-Castelle, D. et Duflo, M. (1993). Probabilités et Statistique. Tome 2. Problèmes à temps mobile. Masson Paris. ISBN 2-225-84373-2. 
[Dette and Studden(1997)] Dette, H. and Studden, W. J. (1997). The theory of canonical moments with applications in statistics, probability, and analysis. John Wiley \& Sons Inc., New York. ISBN 0-47110991-6. A Wiley-Interscience Publication.

[Gamboa and Gassiat(1997)] Gamboa, F. and Gassiat, E. (1997). Bayesian methods and maximum entropy for ill-posed inverse problems. Ann. Statist., 25(1):328-350. ISSN 0090-5364.

[Gamboa et al(1999)] Gamboa, F. , Rouault, A. and Zani, M. (1999). A functional large deviations principle for quadratic forms of Gaussian stationary processes. Stat. \&3 Proba. Letters, 43(1):299-308.

[Gamboa, F. and Lodaza-Chang, L.-V.(2004)] Large deviations for a random power moment problem. Ann. Probab., to appear.

[Gupta, J.C. (1999)] Partial Hausdorff sequences and symmetric probabilities on finite products of $\{0,1\}$. Sankhyā 61, 347-357.

[Karlin, S. and Shapeley, L.S.(1953)] Geometry of moment spaces. Amer. Math. Soc. Memoir No. 12, Amer. Math. Soc., Providence, Rhode Island.

[Karlin and Studden (1966)] Karlin, S. and Studden, W. J. (1966). Tchebycheff systems: With applications in analysis and statistics. Interscience Publishers John Wiley \& Sons, New York-London-Sydney.

[Kreı̆n and Nudelman(1977)] Kreı̆n, M. G. and Nudelman, A. A. (1977). The Markov moment problem and extremal problems. American Mathematical Society, Providence, R.I.. ISBN 0-8218-4500-4. Ideas and problems of P. L. Čebyšev and A. A. Markov and their further development, Translated from the Russian by D. Louvish, Translations of Mathematical Monographs, Vol. 50.

[Landau, H.S.(1987)] Moments in Mathematics. Proc. Symposia in Applied Mathematics 37, AMS, Providence, RI.

[Protter (2004)] Protter, P. E. (2004). Stochastic integration and differential equations. ISBN 3-540-00313-4. Springer. Berlin.

[Najim(2002)] Najim, J. (2002). A Cramér type theorem for weighted random variables. Elec. Jour. of Prob., $4: 1-32$.

[Shohat, J.A. and Tarmarkin, J.D. (1943)] The problem of moments. Mathematical Surveys No. 1, Amer. Math. Soc., Providence, Rhode Island.

[Skibinsky(1967)] Skibinsky, M. (1967). The range of the $(n+1)$-th moment for distributions on [0, 1]. $J$. Appl. Probability, 4:543-552.

[Skibinsky(1968)] Skibinsky, M. (1968). Extreme $n$th moments for distributions on $[0,1]$ and the inverse of a moment space map. J. Appl. Probability, 5:693-701.

[Skibinsky(1969)] Skibinsky, M. (1969). Some striking properties of binomial and beta moments. Ann. Math. Statist., 40:1753-1764.

Mathematik III, NA 3 / 72, Universitätsstra $\beta$ E 150, 44780 Bochum Germany, Laboratoire de Statistiques et de Probabilités, UMR C5583, Université Paul Sabatier, 118 Route de Narbonne 31062 Toulouse cedex 4 France

E-mail address: holger.dette@ruhr-uni-bochum.de

E-mail address: gamboa@math.ups-tlse.fr 